\title{
Fast-track surgery and exclusive enteral nutrition applied to a rat model of heterotopic intestinal transplantation
}

\author{
XINGWEI XU, TAO FENG, XIN GAO, XIN ZHAO, YANNIAN LIAO and WU JI \\ Research Institute of General Surgery, Jinling Hospital, School of Medicine, \\ Nanjing University, Nanjing, Jiangsu 210093, P.R. China
}

Received October 30,2014; Accepted October 27, 2015

DOI: $10.3892 /$ etm.2016.2974

\begin{abstract}
The present study applied fast-track surgery (FTS) concepts and exclusive enteral nutrition (EEN) to a rat model of heterotopic intestinal transplantation (HIT). A total of 96 pairs of Sprague-Dawley rats were randomly distributed into three groups, as follows: i) The conventional group (group 1); ii) the FTS group (group 2); and iii) the FTS with EEN group (EEN group). FTS alterations to the HIT protocol were as follows: i) The use of sevoflurane as an anesthetic; ii) alterations to the order of the procedure and iii) a modified suturing technique. In addition, the EEN group rats underwent an early EEN gavage. The operation time, success rate, recovery state and morphological characteristics of the grafts were compared among the groups. The average operative time was significantly decreased in the group 2 and EEN group rats (137.44 \pm 16.03 and $139.67 \pm 15.25 \mathrm{~min}$, respectively), as compared with the group 1 rats $(169.36 \pm 13.72 \mathrm{~min} ; \mathrm{P}<0.05)$. In addition, the percentage of rats surviving $>14$ days was significantly increased in the group $2(87.5 \%)$ and EEN group $(90.6 \%)$ rats, as compared with the group 1 rats $(68.7 \% ; \mathrm{P}<0.05)$. Furthermore, the villi of graft in EEN group appeared longer, and exhibited narrower interspaces. The ischemia-reperfusion injury and mononuclear cell infiltration were attenuated at postoperative day 7 . The results of the present study suggested that the application of FTS concepts and EEN gavage to HIT may accelerate recovery and ameliorate graft damage following surgery.
\end{abstract}

\section{Introduction}

A successful and classical animal model of heterotopic intestinal transplantation (HIT) was initially described by Monchick and Russell (1) in 1971, and has since been improved. At present, a rat model of HIT is widely used, which has particular value

Correspondence to: $\mathrm{Mr}$. Wu Ji, Research Institute of General Surgery, Jinling Hospital, School of Medicine, Nanjing University, 9 Hankou Road, Nanjing, Jiangsu 210093, P.R. China

E-mail: jiwusky@126.com

Key words: fast-track surgery, intestinal transplantation, rats, exclusive enteral nutrition for use in immunological studies. The majority of technical difficulties associated with this model have been surmounted; however, the present study hypothesized that the rat model of HIT may be improved by the application of fast-track surgery (FTS) concepts and exclusive enteral nutrition (EEN) gavage.

FTS comprises a combination of perioperative interventions, which aim to reduce postoperative stress, the incidence of postoperative complications, and the length of hospital stays (2). In particular, FTS consists of revised traditional surgical practices, improved anesthesia and minimized surgical stress, which have proved effective in major human surgical procedures since the introduction of FTS in the early 1990s $(3,4)$. EEN is a nutritional therapy used for inducing remission in patients with Crohn's disease, and involves a period of 6-8 weeks, during which the patient subsists on an exclusively liquid diet, consisting of either elemental or polymeric formulae $(5,6)$. During this period, the patients are not allowed to consume other dietary items, with the exception of water and various beverages $(5,6)$. To the best of our knowledge, the application of EEN gavage to a rat model of HIT has yet to be reported. The present study hypothesized that EEN gavage may help to stabilize the structure of the mucosal barrier, re-establish the gut bacterial environment and ameliorate the survival rate in a rat model of HIT.

\section{Materials and methods}

Rats and experimental design. A total of 192 male Sprague-Dawley rats (weight, 250-330 g; age, 8-9 weeks) from the Department of Comparative Medicine, Jinling Hospital (Nanjing, China), underwent HIT. The rats were housed in rodent facilities, and were maintained on a $12 \mathrm{~h}$ light/dark cycle, with ad libitum access to commercially available chow and tap water. The temperature was maintained at $\sim 20^{\circ} \mathrm{C}$. Surgical procedures were conducted by two junior microsurgeons. Rat care protocols and experiments were conducted in accordance with the Principles of Laboratory Animal Care and the Guide for the Care and Use of Laboratory Animals (National Institutes of Health, Bethesda, MA, USA), and were designed according to the Rules for the Medical Laboratory Animal, as published by the Ministry of Health of the People's Republic of China. The present study was approved by the Ethics Committee of Jinling Hospital, and Institutional Review Board approval was obtained. 


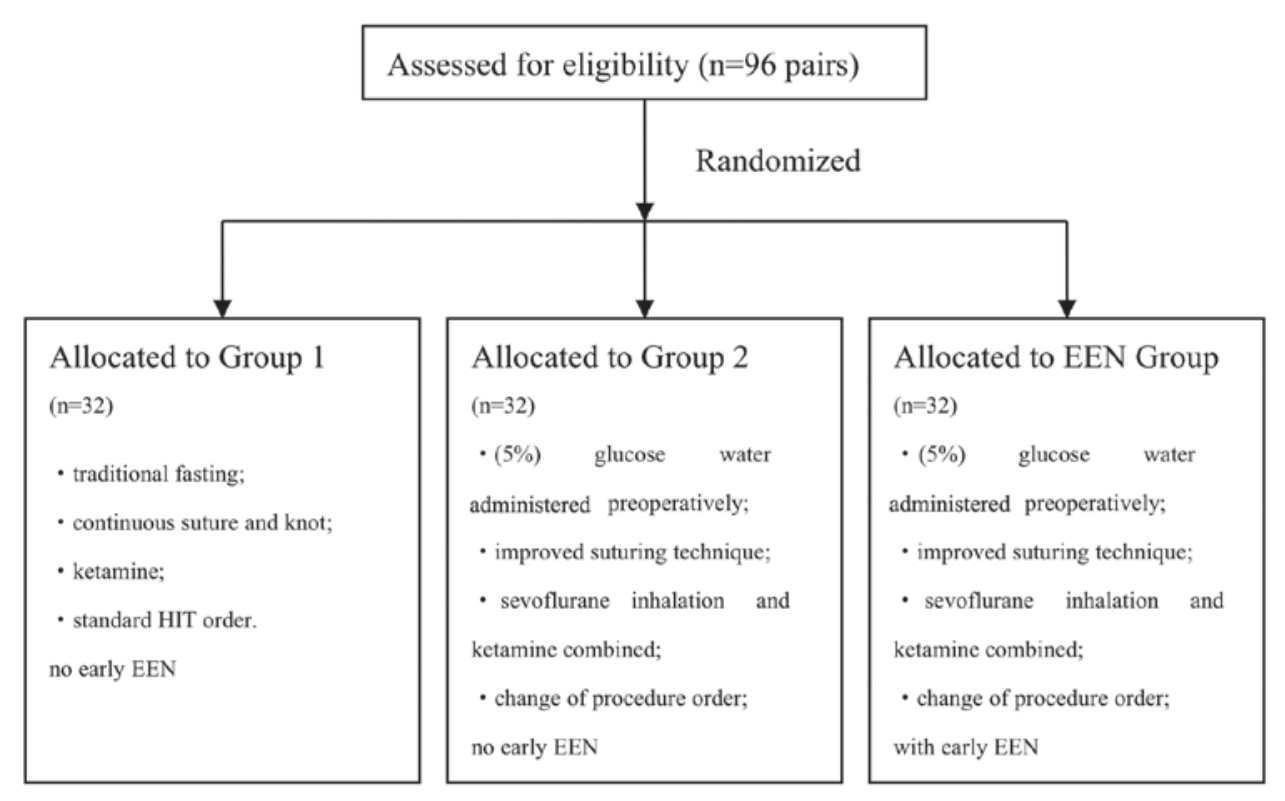

Figure 1. Allocation of rats throughout the study. HIT, heterotopic intestinal transplantation; EEN, exclusive enteral nutrition.
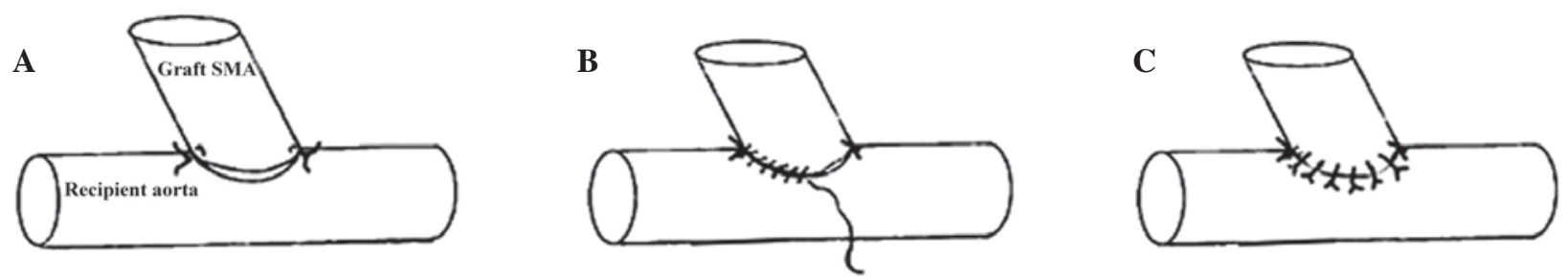

Figure 2. Schematic representation of novel suturing technique. (A) Arteries from the donor and recipient rats were anastomosed using a 10-0 suture consisting of two knots, one placed at the proximal apex of the anastomosis and the other at the distal apex. (B) Simple continuous suture from the distal apex of the anastomosis in a counterclockwise direction without knotting, loosening the suture. (C) Each part of the suture was cut and then knotted. Five or six knots were needed for each side and knots were added according to the requirement.

The rats were randomly divided into 96 pairs, including a donor and recipient rat, and were distributed into three groups ( $\mathrm{n}=32 /$ group): i) The conventional group (group 1); ii) the FTS group (group 2); and iii) the FTS with EEN group (EEN group; Fig. 1). For group 1 rats, the protocols were consistent with the standard and perioperative care procedures outlined in a previous study (5). For group 2 rats, various modifications were made according to FTS concepts, including preoperative treatment with glucose water $(5 \%)$, the use of sevoflurane inhalation anesthesia, and alterations to the surgical procedure order and suturing technique. In addition, for the EEN group, an early EEN gavage was conducted. The rats were not treated with any immunosuppressive therapies postoperatively.

\section{Interventions}

Anesthesia. The rats in group 1 were anesthetized with an intraperitoneal injection of ketamine $(100 \mathrm{mg} / \mathrm{kg}$; Jiangsu Hengrui Medicine Co. Ltd., Lianyungang, China) dissolved in saline. In addition, $50 \mathrm{mg} / \mathrm{kg}$ ketamine was administered perioperatively when required. The rats in group 2 and the EEN group were anaesthetized with sevoflurane via a self-made plastic bottle containing gauze saturated in sevoflurane (Baxter International, Inc., Deerfield, IL, USA). Anesthesia was initiated and induced by placing the bottle over the head of the rats. Following the initial anesthetization, $60 \mathrm{mg} / \mathrm{kg}$ ketamine was administered in order to achieve an adequate depth of anesthetization. Anesthesia was maintained throughout the surgery by administering sevoflurane every $20 \mathrm{~min}$. The gauze was renewed in order to maintain the desired concentration of sevoflurane in the bottle.

Fasting. All rats in group 1 received normal food and water up to $8 \mathrm{~h}$ prior to surgery. Conversely, the rats in group 2 and the EEN group had ad libitum access to glucose water (5\%) during the fasting period.

Suturing technique. An end-to-side anastomosis was performed between the aortic patch of the donor rat and the abdominal aorta of the recipient rat. In group 1, a conventional continuous suture was used, as outlined in a previous study (5). Conversely, in group 2 and the EEN group, the arteries of the donor and recipient rats were initially anastomosed using a 10-0 suture (Double Arrow; Yiling Medical Device Sales Co. Ltd., Shanghai, China) consisting of two knots, one placed at the proximal apex and the other at the distal apex of the anastomosis. Subsequently, continuous 10-0 suturing was performed at the posterior and anterior walls, using an 'out-in/in-out' technique, from the graft to the recipient. Without tightening the suture, each part of the suture was cut and knotted ( 5-6 knots were needed for each side and additional knots were added when required; Fig. 2). 
HIT technique. In group 2 and the EEN group, the recipient rats were prepared prior to completing the final irrigation step to obtain the donor graft. Briefly, following dissection of the left renal vein of the recipient rat, the intrarenal abdominal aorta was carefully handled, and a moist gauze was used to cover the surgical area in preparation for the graft. Subsequently, the donor rat was returned to for the final irrigation step.

Early EEN. In the EEN group, the recipient rats underwent EEN by gavage, during which the rats received the standard total calories per day for rats $(836.8 \mathrm{~kJ} ; 200 \mathrm{kcal}) /(\mathrm{kg}$ x day). The enteral formula used in the present study was the commercially available Peptisorb Liquid (Nutricia Research, Amsterdam, Netherlands), which consists of maltodextrin, hydrolyzed whey protein peptide, fat, vitamins and trace elements. A summary of the composition of Peptisorb Liquid is presented in Table I (6). The dosage and gavage speed were gradually increased from half ( $6 \mathrm{~h}$ post-surgery) to full strength (at postoperative day 1), in order to reduce side effects, including diarrhea and vomiting. Throughout this period, the rats in the EEN group were not allowed to consume other dietary items, with the exception of water. Groups 1 and 2 rats received a normal diet of chow.

Morphological alterations to the intestinal mucosa. Morphological alterations to the graft intestinal mucosa were detected using light microscopy (BX60; Olympus Corporation, Tokyo, Japan), according to a common protocol (6). The length of the intestinal villus was measured using cartographic software at various time points $(12,24$ and 48 h) (7). Graft pathological examination was conducted on postoperative day 7. The allografts were collected from a stoma each time. The rats were sacrificed by cervical dislocation.

Statistical analysis. Statistical analyses were conducted using SPSS software 12.0 (SPSS, Inc., Chicago, IL, USA). Data are presented as the mean \pm standard deviation. Comparisons between groups were conducted using one-way analysis of variance, followed by Dunnett's multiple comparisons test. Comparisons between two groups were conducted using paired and unpaired t-tests. $\mathrm{P}<0.05$ was considered to indicate a statistically significant difference.

\section{Results}

Response of rats to HIT. A total of 96 pairs of rats underwent HIT by a single group of surgeons between September 2012 and May 2013. The majority of procedures were successful and the rats appeared to tolerate HIT well. The rats exhibited a good appetite upon awakening from anesthesia and were in good health. No significant differences in the overall conditions of the rats, including body weight and baseline characteristics, were detected among the three groups prior to surgery.

Effects of interventions on HIT procedure. The surgical duration for each group is presented in Table II. As the procedure order was altered in group 2 and the EEN group, only the total surgery time was calculated. The surgical time was significantly decreased in group 2 and the EEN group rats $(137.44 \pm 16.03$ and $139.67 \pm 15.25 \mathrm{~min}$, respectively), as compared with the group 1 rats $(169.36 \pm 13.72 \mathrm{~min} ; \mathrm{P}<0.05)$.
Table I. Constituents of Peptisorb liquid (energy, 500 kcal per $500 \mathrm{ml})$.

\begin{tabular}{|c|c|}
\hline Component & Mass (mg) \\
\hline Maltodextrin & 88,000 \\
\hline Hydrolyzed whey protein peptide & 20,000 \\
\hline Lipid (vegetable oil) & 8,500 \\
\hline Potassium & 750 \\
\hline Sodium & 500 \\
\hline Calcium & 400 \\
\hline Magnesium & 115 \\
\hline Phosphorus & 360 \\
\hline Chlorine & 625 \\
\hline Iron & 8.0 \\
\hline Zinc & 6.0 \\
\hline Copper & 0.9 \\
\hline Manganese & 1.65 \\
\hline Fluorine & 0.5 \\
\hline Iodine & 0.065 \\
\hline Molybdenum & 0.05 \\
\hline Chromium & 0.0335 \\
\hline Selenium & 0.0285 \\
\hline Vitamin A & 0.41 \\
\hline Vitamin B1 & 0.75 \\
\hline Vitamin B2 & 0.8 \\
\hline Vitamin B6 & 0.85 \\
\hline Vitamin B12 & 0.00105 \\
\hline Vitamin C & 50.0 \\
\hline Vitamin D & 0.0035 \\
\hline Vitamin E & 6.5 \\
\hline Vitamin K & 0.0265 \\
\hline Niacin & 9.0 \\
\hline Pantotenic acid & 2.65 \\
\hline Folic acid & 0.135 \\
\hline Biotin & 0.02 \\
\hline Choline & 185 \\
\hline Carotenoids mixture & 1.0 \\
\hline Taurine & 0.05 \\
\hline
\end{tabular}

In group 1 , the average recovery time from anesthesia was $\sim 25.64 \pm 9.45 \mathrm{~min}$, which was significantly increased, as compared with the group 2 and EEN group rats $(\mathrm{P}<0.05)$.

The incidence of complications frequently associated with HIT was compared among the groups, and are presented in Table III. Hemorrhagic shock, in particular uncontrollable bleeding in the arterial anastomotic area, was the complication most frequently associated with HIT failure, and the rats typically succumbed to surgery. Two cases of hemorrhagic shock occurred in group 1, but were absent from the other groups. One case of an anesthetic accident occurred in group 2, and was associated with excessive inhalation of sevoflurane. The surgical success rate at 3 days post-surgery was $84.4 \%$ for group 1, 93.8\% for group 2 and $96.9 \%$ for the EEN group rats. In addition, comparing the 14-day survival rate indicated that 
Table II. Duration of surgery (min) for each group.

\begin{tabular}{ccccc}
\hline Group & Donor part & Recipient part & Total surgery time & Awakening time \\
\hline 1 & $59.45 \pm 8.16$ & $109.91 \pm 8.21$ & $169.36 \pm 13.72^{\mathrm{a}}$ & $25.64 \pm 9.45^{\mathrm{a}}$ \\
2 & N/A & N/A & $137.44 \pm 16.03$ & $14.81 \pm 4.12$ \\
EEN & N/A & N/A & $139.67 \pm 15.25$ & $15.11 \pm 4.06$ \\
\hline
\end{tabular}

The surgical duration and awakening time following anesthesia in group 1 were significantly longer, as compared with group 2 and the EEN group $\left({ }^{\mathrm{P}} \mathrm{P}<0.05\right)$. EEN, exclusive enteral nutrition; N/A, not applicable.

Table III. Perioperative complications leading to mortality.

\begin{tabular}{lccc}
\hline Complications & Group 1 & Group 2 & EEN group \\
\hline Hemorrhagic shock & 2 during surgery & - & - \\
Anesthetic accident & - & 1 during surgery & - \\
Portal vein thrombosis or stenosis & 2 in 3 days & 1 in 3 days & 1 in 3 days \\
Intestinal obstruction & 1 in 3 days & - & - \\
Survival at 3 days & $27(84.4 \%)$ & $30(93.8 \%)$ & $31(96.9 \%)$ \\
Infection of abdominal cavity & 2 in 14 days & 1 in 14 days & - \\
Other complications & 3 in 3 days & 1 in 14 days & 2 in 14 days \\
Survival at 14 days & $24(68.7 \%)$ & $28(87.5 \%)$ & $29(90.6 \%)^{\mathrm{a}}$
\end{tabular}

$\mathrm{n}=32$ /group. The 14 day survival rate was significantly increased in the EEN group, as compared with group 1 ( $\left.{ }^{\mathrm{a}}<0.05\right)$. EEN, exclusive enteral nutrition.

there was a statistically significant difference between the EEN group and group $1(\mathrm{P}<0.05)$; the 14-day survival rate was $68.7 \%$ for group 1, $87.5 \%$ for group 2 and $90.6 \%$ for the EEN group rats.

Morphological alterations of the intestinal mucosa. Grafts were obtained from all rats in order to evaluate histological alterations at specific time points. Light microscopy demonstrated that the intestinal villi of the grafts from all three groups gradually became eroded, necrotic and were decomposed following HIT, and these lesions were most severe at postoperative day 1. By the end of the study, the villi from the EEN group rats exhibited the least severe morphological alterations under a transmission electron microscope, and the villi were significantly longer and exhibited narrower interspaces, as compared with the group 1 and 2 rats $(\mathrm{P}<0.01$; Fig. 3). Histological examination of the grafts using hematoxylin and eosin staining on day 7 detected less severe ischemia-reperfusion injury and markedly decreased mononuclear cell infiltration, cryptitis and rupture in the tips of the villi from the EEN group grafts, as compared with the group 1 and 2 grafts (Fig. 4).

\section{Discussion}

Intestinal transplantation has emerged as a potential strategy for the treatment of end-stage intestinal failure, and remains essential for the investigation of immunology and the underlying mechanisms of graft rejection in animal models (8). Complications exist regarding the complex microvascular techniques and high mortality rates associated with HIT. The present study reports the use of a novel HIT protocol, in which FTS concepts and postoperative EEN gavage were applied. These have previously been associated with beneficial effects in recovering patients in numerous clinical trials (9-14). In particular, the present study compared the success rate, graft conditions and survival outcomes of the novel vs. traditional methods in a rat model of HIT.

In a pre-test (data not shown), controlling the dose of perioperatively administered ketamine was shown to be challenging, and was frequently associated with respiratory failure or cardiac arrest. Therefore, sevoflurane anesthesia, which is widely used in clinical practice due to its safe induction, rapid emergence, hemodynamic stability and nonirritating airway properties, was selected for the present study. In addition, decreasing the amount of ketamine decreased the frequency of writhing times and improved the sleeping state of the rats. Additional ketamine has previously been shown to decrease the incidence of emergence agitation, and exert analgesic and hypnotic effects following sevoflurane general anesthesia (15). In the novel HIT method established in the present study, the rats who awoke perioperatively returned to sleep immediately upon additional treatment with sevoflurane, and each successive treatment lasted for $\sim 20 \mathrm{~min}$. The rats in group 2 and the EEN group exhibited rapid recovery from surgery and improved flexibility, as compared with the rats in group 1, and this was not associated with adverse effects on the long-term survival rates of the rats. A short recovery time following anesthesia is beneficial for the survival rate, since it may help to avoid low body temperature. These results suggested that 


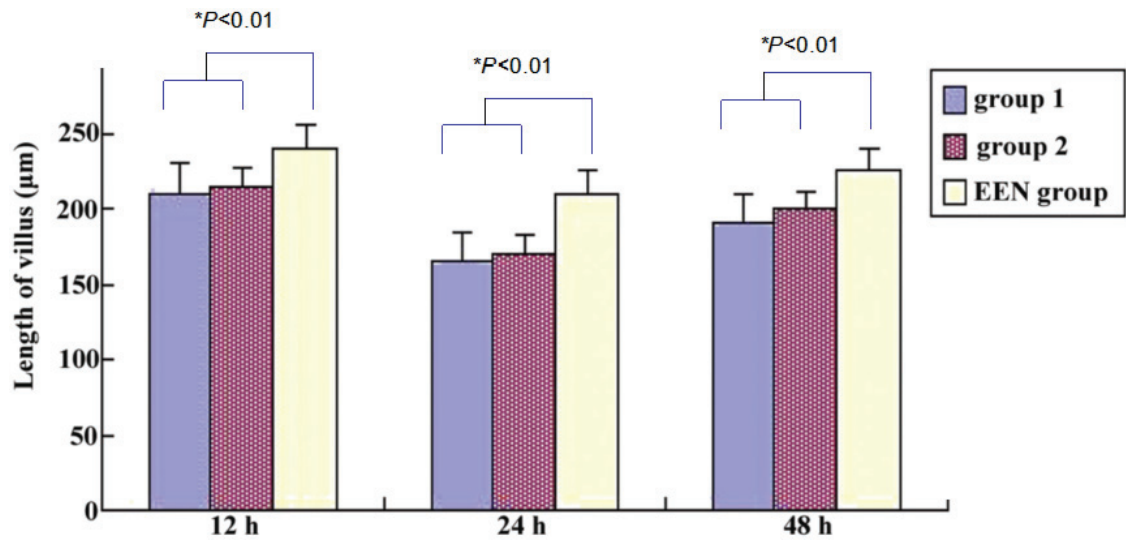

Figure 3. Quantification of villus length in the group 1, group 2 and EEN group rats. Rats subjected to HIT exhibited severe lesions on the intestinal mucosa at $48 \mathrm{~h}$ post-surgery and shortened villus length. Postoperative EEN was able to significantly attenuate the effects of HIT on the villi of the EEN group rats, as compared with the group 1 and 2 rats. Data are presented as the mean \pm standard error of the mean. ${ }^{*} \mathrm{P}<0.01$ vs. group 1 and 2 . EEN, exclusive enteral nutrition; HIT, heterotopic intestinal transplantation.
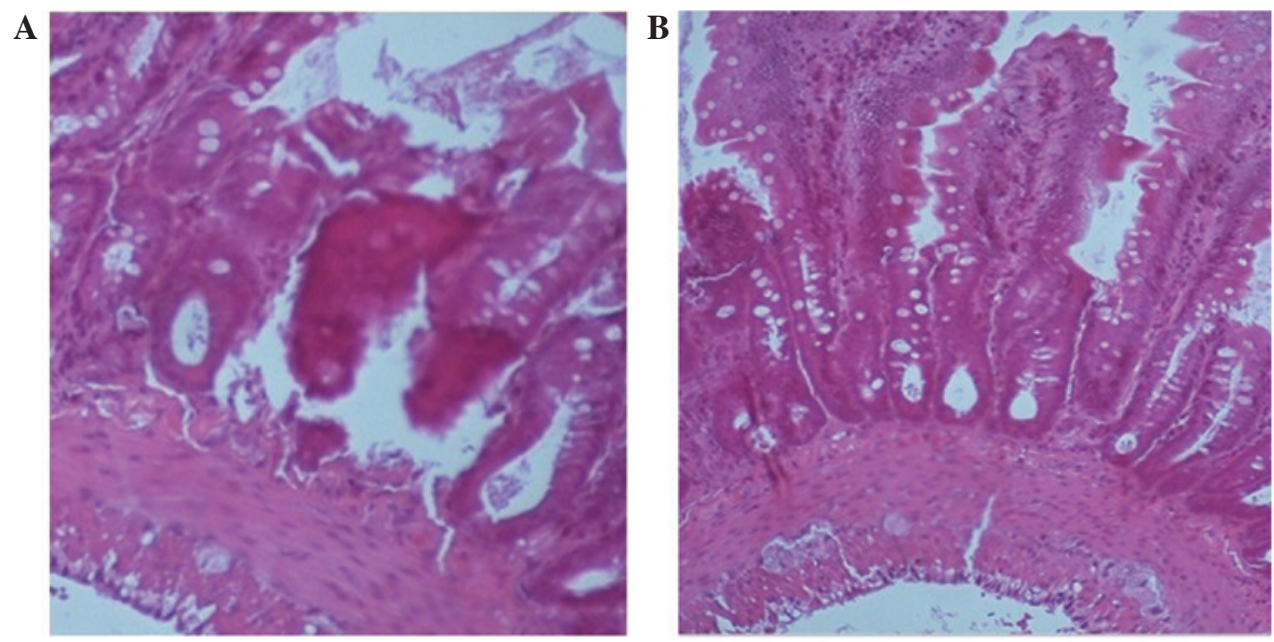

Figure 4. Hematoxylin and eosin staining of the grafts 7 days following transplantation (magnification, x10). (A) Histological morphology of the syngeneic graft from the group 1 rats at postoperative day 7. The graft exhibited serious ischemia-reperfusion injury and severe mononuclear cell infiltration, cryptitis and rupture in the tips of the villi. (B) Histological morphology of the graft from the EEN group rats at day 7 following HIT was markedly improved, as compared with group 1. EEN, exclusive enteral nutrition; HIT, heterotopic intestinal transplantation.

the combined use of sevoflurane and ketamine was an ideal strategy for anesthetizing recipient rats for HIT.

Arterial anastomosis is the major procedure in HIT and several months of training is required in order to gain competence in the technique. Previous studies have reported conflicting results regarding the use of an interrupted or continuous suturing technique in end-to-side anastomosis (16). The novel suturing technique used in the present study combined the advantages of both techniques. In particular, it was time-saving without increasing the possibility of bleeding. Wang et al (16) reported that bleeding typically occurs from within the inter-stitch space during the suturing process, particularly near to the proximal and distal points; thus suggesting that controlling the size of the inter-stitch space is required in order to reduce blood loss. This was easy to achieve using the novel suturing method, in which each part of the continuous suture was cut and knotted. Therefore, the alteration to the suturing procedure may benefit the training process and save time.
Ideally, the cold ischemia time should be as short as possible: Kato et al (17) demonstrated that prolonged cold ischemia/reperfusion enhanced the rate of immune rejection and affected the recovery of bowel function. In the group 2 and EEN group rats, the procedure order was altered in an attempt to reduce the impact of cold ischemia. In particular, various steps of the recipient preparation were completed ahead of the graft lavage with the aid of an assistant, including isolation of the recipient artery and renal vein. Initially, there was concern regarding whether there would be an increased risk of rat mortality associated with the extended duration of anesthesia; however, the altered protocol effectively reduced cold ischemia time when using the novel method of sevoflurane inhalation anesthesia.

Nutritional support is necessary for HIT patients, as it improves nutrient absorption and limits food antigen overload, which may trigger immune stimulation and a subsequent increased risk of acute graft rejection (18). Glucose water (5\%) is rich in sugar and energy, and its preoperative use in the 
present study helped the recipient rats to tolerate the long surgical duration. Previous studies suggested that enteral nutrition may also promote the reconstruction of mucosa, improve impaired intestinal transit and regulate immune functions (19). In addition, Zhang et al (20) reported that enteral rehabilitative therapy was able to induce potent trophic effects on the graft structure and recipient metabolism. The underlying mechanism by which the diet may exert such beneficial effects is currently unclear; however, an arginine and glutamine deficiency has previously been shown to initiate immune function decline and inflammatory responses $(6,7)$. The Nutrison Fibre used in the present study contained numerous amino acids, which served as an essential supplement and protected the intestinal barrier function. The European Society for Clinical Nutrition and Metabolism guidelines for enteral nutrition postoperatively recommend that enteral feeding is initiated within $24 \mathrm{~h}$ following surgery, since a previous study demonstrated that early enteral nutrition decreased the rate of postoperative complications due to infection, and the duration of hospitalization (21). In the EEN group, EEN gavage was conducted immediately post-surgery and lasted for $>3$ days, and was associated with an increased survival rate and improved graft conditions.

In conclusion, the results of the present study suggested that the application of FTS concepts and EEN gavage to a rat model of HIT was able to improve the recovery and graft function of the recipient rats. Future studies should investigate the molecular mechanisms underlying EEN therapy, in order to reduce the risk of graft rejection, improve gut trophicity and optimize nutrient absorption. The perioperative interventions applied in the present study may improve HIT modeling and reduce the suffering of the experimental rats.

\section{Acknowledgements}

The authors of the present study would like to thank B Shen, Y Xu and Q Zhao (Jinling Hospital, Nanjing University, Jiangsu, China) for their helpful suggestions and assistance throughout the present study and for preparing the manuscript. The present study was supported by grants from the Natural Science Foundation of Jiangsu Province (grant no. BK2008237) and the Nanjing Science and Technology Development Project (grant no. 201104027).

\section{References}

1. Monchik GJ and Russell PS: Transplantation of small bowel in the rat: Technical and immunological considerations. Surgery 70 : 693-702, 1971

2. Serclová Z, Dytrych P, Marvan J, Nová K, Hankeová Z, Ryska O, Slégrová Z, Buresová L, Trávníková L and Antos F: Fast-track in open intestinal surgery: Prospective randomized study (Clinical Trials Gov Identifier no. NCT00123456). Clin Nutr 28: 618-624, 2009.

3. Slim K: Fast-track surgery: The next revolution in surgical care following laparoscopy. Colorectal Dis 13: 478-480, 2011.
4. Lassen K, Soop M, Nygren J, Cox PB, Hendry PO, Spies C, von Meyenfeldt MF, Fearon KC, Revhaug A, Norderval S, et al; Enhanced Recovery After Surgery (ERAS) Group: Consensus review of optimal perioperative care in colorectal surgery: Enhanced Recovery After Surgery (ERAS) Group recommendations. Arch Surg 144: 961-969,2011.

5. Wu XT, Li JS, Zhao XF, Zhuang W and Feng XL: Modified techniques of heterotopic total small intestinal transplantation in rats. World J Gastroenterol 8: 758-762, 2002.

6. Feng Y, Li Y, Mei S, Zhang L, Gong J, Gu L, Zhang W, Zhu W, Li N and Li J: Exclusive enteral nutrition ameliorates mesenteric adipose tissue alterations in patients with active Crohn's disease. Clin Nutr 33: 850-858, 2014.

7. Li Y, Chen Y, Zhang J, Zhu JF, Liu ZJ, Liang SY, Sun K, Liao WY and Gong JP: Protective effect of glutamine-enriched early enteral nutrition on intestinal mucosal barrier injury after liver transplantation in rats. Am J Surg 199: 35-42, 2010.

8. Grant D, Abu-Elmagd K, Reyes J, Tzakis A, Langnas A, Fishbein T, Goulet $\mathrm{O}$ and Farmer D; Intestine Transplant Registry: 2003 report of the intestine transplant registry: A new era has dawned. Ann Surg 241: 607-613, 2005.

9. Basse L, Jakobsen DH, Bardram L, Billesbølle P, Lund C, Mogensen T, Rosenberg J and Kehlet H: Functional recovery after open versus laparoscopic colonic resection: A randomized, blinded study. Ann Surg 241: 416-423, 2005.

10. King PM, Blazeby JM and Ewings P: The influence of an enhanced recovery programme on clinical outcomes, costs and quality of life after surgery for colorectal cancer. Colorectal Dis 8: 506-513, 2006.

11. Gatt M, Anderson AD, Reddy BS, Hayward-Sampson P, Tring IC and MacFie J: Randomized clinical trial of multimodal optimization of surgical care in patients undergoing major colonic resection. Br J Surg 92: 1354-1362, 2005.

12. Khoo CK, Vickery CJ, Forsyth N, Vinall NS and Eyre-Brook IA: A prospective randomized controlled trial of multimodal perioperative management protocol in patients undergoing elective colorectal resection for cancer. Ann Surg 245: 867-872, 2007.

13. Muller S, Zalunardo MP, Hubner M, Clavien PA and Demartines N; Zurich Fast Track Study Group: A fast-track program reduces complications and length of hospital stay after open colonic surgery. Gastroenterology 136: 842-847, 2009.

14. Serclová Z, Dytrych P, Marvan J, Nová K, Hankeová Z, Ryska O, Slégrová Z, Buresová L, Trávníková L and Antos F: Fast-track in open intestinal surgery: prospective randomized study (Clinical Trials Gov Identifier no. NCT00123456). Clin Nutr 28: 618-624, 2009.

15. Abu-Shahwan I and Chowdary K: Ketamine is effective in decreasing the incidence of emergence agitation in children undergoing dental repair under sevoflurane general anesthesia. Paediatr Anaesth 17: 846-850, 2007.

16. Wang J, Ma H, Zhang H, Lu B, Wang J, Wang Z, Li Y and Li J: Continuous locked suture technique for arterial anastomosis in rat small bowel transplantation. Microsurgery 27: 112-117, 2007.

17. Kato T, Tzakis AG, Selvaggi G, Gaynor JJ, David AI, Bussotti A, Moon JI, Ueno T, DeFaria W, Santiago S, et al: Intestinal and multivisceral transplantation in children. Ann Surg 243: 756-766, 2006.

18. Colomb V and Goulet O: Nutrition support after intestinal transplantation: How important is enteral feeding? Curr Opin Clin Nutr Metab Care 12: 186-189, 2009.

19. Hegazi RA and O'Keefe SJ: Nutritional immunomodulation of acute pancreatitis. Curr Gastroenterol Rep 9: 99-106, 2007.

20. Zhang XQ, Li JS, Li N, Li YS and Fan XH: Trophic effect of enteral rehabilitative therapy in rat small bowel transplantation. Transplant Proc 37: 2351-2353, 2005.

21. Weimann A, Braga M, Harsanyi L, Laviano A, Ljungqvist O, Soeters P; DGEM (German Society for Nutritional Medicine), Jauch KW, Kemen M, Hiesmayr JM, et al; ESPEN (European Society for Parenteral and Enteral Nutrition): ESPEN Guidelines on Enteral Nutrition: Surgery including organ transplantation. Clin Nutr 25: 224-244, 2006. 